\title{
AUS DER PRAXIS
}

\section{Die zukünftigen Aufgaben des Staates}

\author{
Martin Beckmann \\ Wolfgang Uellenberg-van Dawen
}

\section{Ausgehend von den Immobilienmärkten in den Vereinigten Staaten hat sich zuerst \\ KRISE DES WETTBEWERBSSTAATS UND NEUES LEITBILD} eine Krise des globalen Finanzsektors und schließlich eine dramatische Weltwirtschaftskrise entwickelt. Sie ist jedoch nicht nur eine Krise des globalen Finanzmarktkapitalismus und der mit ihm verbundenen neoliberalen Wirtschaftsideologie. Die Orientierung staatlicher Politik an den internationalen Kapitalmärkten und der Wandel des Sozialstaates zu einem an globalen Märkten orientierten Wettbewerbsstaat sind grundsätzlich infrage gestellt.

In der derzeitigen Situation eröffnet sich die Möglichkeit, ein neues Leitbild von Staatlichkeit, das sich am Sozialstaatsgebot des Grundgesetzes bzw. der Europäischen Sozialcharta orientiert, zu entwickeln und durchzusetzen, in dem die Dimension des Öffentlichen gegenüber dem Privaten wieder an Bedeutung gewinnt. Diese angestrebte Wiederbelebung des Öffentlichen umfasst Formen staatlicher Regulierung und Kontrolle des Finanzsektors, verstärkte Infrastrukturinvestitionen, den Ausbau öffentlicher Dienstleistungen und die Stärkung der sozialen Sicherheit. Gegen die Tendenz der letzten Jahrzehnte, soziale Infrastruktur und soziale Sicherheit zu privatisieren und der Dynamik der Kapitalmärkte auszuliefern, soll dem Sozialstaatsangebot des Grundgesetzes ${ }^{1}$ wieder größeres Gewicht gegeben und menschenwürdige Arbeits- und Lebensbedingungen müssen gewährleistet werden. Dabei müssen auch die Fehlentwicklungen sozialstaatlicher Leistungserbringung und dadurch verursachte Qualitätsverluste reflektiert werden. Schließlich muss eine neue Politik aktiven sozialstaatlichen Handelns auf mehreren Ebenen regional, national, europäisch sowie global entwickelt und durchgesetzt werden. Dies ist nur möglich, wenn der soziale Zusammenhang der Gesellschaft erneuert wird sowie Solidarität und soziale Gerechtigkeit gestärkt werden.
Die Profitabilitätskrise des Kapitals setzte seit den frühen 1970er Jahren den keynesianischen Wohlfahrtsstaat unter Druck. Die Multinationalisierung großer Unternehmen als ökonomische und die Aufhebung des Bretton-Woods-Systems durch die von Richard Nixon geführte US-Administration als politische Reaktion deuteten an, dass eine Ära zu Ende ging und sich damit auch das Leitbild von Staatlichkeit wandelte. Zurückgehende Wachstumsraten, der Anstieg der Arbeitslosigkeit und die wachsende Inflation erhöhten den Legitimationsdruck auf die keynesianischen Wohlfahrtsstaaten.

In dieser Situation gewannen neoliberale Konzepte in den Expertendiskursen ebenso wie in der medialen Debatte an Einfluss. Die Wahlsiege von Margaret Thatcher (Großbritannien, 1979) und Ronald Reagan (USA, 1981) markierten endgültig den Übergang zu einer neuen Staatlichkeit, dem liberalen Wettbewerbsstaat. Dieser setzte auf die Entfesselung der Marktkräfte durch Privatisierung öffentlicher Unternehmen und sozialer Sicherungssysteme, Liberalisierung von Finanz- und Gütermärkten und Deregulierung des Arbeitsmarktes. Und so wie der keynesianische Wohlfahrtsstaat international über das Bretton-Woods-System abgesichert wurde, so unterstützen globale und europäische Regeln die neoliberale Politik. Während der Einfluss von globalen Organisationen wie Weltbank oder Internationaler Währungsfonds (IWF) über Strukturanpassungsprogramme vornehmlich für die Staaten des globalen Südens von Bedeutung ist, so hat in Europa der Strukturwandel der europäischen Integration zu einer europäischen Dimension liberaler Wettbewerbsstaatlichkeit geführt. $^{2}$

Mit dem EG-Binnenmarkt und der von den Institutionen der EU hartnäckig ver- folgten Durchsetzung der sogenannten Grundfreiheiten für Kapital, Güter, Dienstleistungen und Arbeitskräfte wurden immer weitere Bereiche der öffentlichen Daseinsvorsorge und Daseinsfürsorge den Wettbewerbsprinzipien unterworfen und damit kommunaler, regionaler und nationaler demokratisch legitimierter Kontrolle und Gestaltung entzogen. Schreitet so die negative Integration der EU voran, so bleibt die positive Integration sozialstaatlicher Standards zur Verhinderung von Lohn- und Sozialdumping und eines negativen Wettlaufs um niedrige Steuersätze weit hinter den Notwendigkeiten eines europäischen Sozialmodells zurück. Im Gegenteil: Der EG-Binnenmarkt hat die destruktive Dynamik deregulierter Märkte, insbesondere der Finanzmärkte, noch verstärkt. Im Zuge der Einführung des Euro schuf man mit der Europäischen Zentralbank (EZB) eine von politischen Weisungen unabhängige Institution, die nur der Preisstabilität verpflichtet ist. Und der Europäische Gerichtshof (EuGH) hat mit verschiedenen Urteilen die Geltung von Streikrecht und Tarifautonomie unter den Vorbehalt der Vereinbarkeit mit den Binnenmarktprinzipien der Dienstleistungsund Niederlassungsfreiheit gestellt. Die in

\footnotetext{
1 Abendroth, W. (1966): Das Grundgesetz. Eine Einführung in seine politischen Probleme, Pfullingen. 2 Ziltener, P. (1999): Strukturwandel der europäischen Integration, Münster.
}

Martin Beckmann, Dr., ist Referent bei der Bundesverwaltung der Vereinten Dienstleistungsgewerkschaft (ver.di) im Bereich Politik und Planung. e-mail: Martin.Beckmann@verdi.de Wolfgang Uellenberg-van Dawen, Dr., ist Bereichsleiter Politik und Planung bei der Bundesverwaltung der Vereinten Dienstleistungsgewerkschaft (ver.di). e-mail: Wolfgang.Uellenberg@verdi.de 
den Mitgliedstaaten der EU nach 1945 bzw. in Mittel- und Osteuropa nach 1990 erkämpften politischen Grundrechte müssen sich so zunehmend gegen die „Freiheitsrechte" deregulierter Märkte behaupten. Das Demokratiedefizit der EU wird immer offenkundiger. Die globale Finanzmarktkrise führt den Mitgliedstaaten der EU und den europäischen Institutionen drastisch vor Augen, dass dieses Defizit staatlicher Regulierung und demokratischer Legitimierung der $\mathrm{EU}$ dringend beseitigt werden muss.

Der Sozialstaat wird seine aktuellen wie seine künftigen Aufgaben nur dann lösen können, wenn er sich von der wettbewerbsstaatlichen und kapitalmarktorientierten Politik abwendet und wieder seinen Aufgaben als aktiver, solidarischer und sozial gerechter Sozialstaat zuwendet.

Wir treten für einen aktiven Staat ein, der die Wirtschaft reguliert, Innovationen und Investitionen fördert, für eine qualitativ hochwertige Infrastruktur sorgt, ein ökologisch verträgliches und nachhaltiges Wachstum fördert und damit die Binnennachfrage stärkt und so aktiv die Arbeitslosigkeit bekämpft. Wir wollen einen solidarischen Staat, der die Beiträge aller für das Gemeinwohl einfordert und der darauf setzt, dass die Starken den Schwachen, die Gesunden den Kranken und die Generationen einander helfen. Und wir wollen einen sozial gerechten Staat, der für eine gerechte Besteuerung und aktuell für eine gerechte Verteilung der Lasten der Krise sorgt. Wir wollen einen handlungsfähigen, aber keinen starken Staat. Er soll nicht die Menschen und ihre Freiheitsrechte einengen, sondern durch Solidarität und soziale Gerechtigkeit Freiheitsräume erst schaffen.

\section{REGULIERUNG DER KAPITALMÄRKTE}

Eine wesentliche zukünftige Aufgabe staatlichen Handelns ist die aktive Beschränkung der herausragenden Rolle, die die Finanzmärkte in den letzten Jahrzehnten gespielt haben. Wir wollen nicht nur Rettungsmaßnahmen, sondern eine grundlegende Wende: Es geht darum, dass sich die Politik vom Primat der globalen Finanzmärkte befreit und wieder selbst das Heft in die Hand nimmt. Dazu ist eine verschärfte Regulierung des Finanzsektors unverzichtbar.

Sämtliche Finanzinstitute, ob Zweckgesellschaften, Private-Equity-Fonds oder Rating-Agenturen, müssen staatlich kon- trolliert werden. Bestimmte hochspekulative Einrichtungen wie Hedge-Fonds sollten komplett verboten und Offshore-Zentren geschlossen werden. Auch müssen künftig alle Finanzprodukte einer staatlichen Bewilligungspflicht unterliegen. Damit kann insbesondere der Derivatemarkt besser kontrolliert werden. Außerdem sollten kurzfristige Finanztransaktionen durch die Tobin-Steuer zumindest erheblich teurer werden. Die Shareholder-Value-Orientierung vieler Unternehmen ging zulasten der Beschäftigten und muss durch eine an langfristigen Unternehmenszielen - sprich: qualitativ hochwertige Güter und Dienstleistungen sowie gute Arbeitsbedingungen - orientierte Entwicklung ersetzt werden. Schließlich muss in der EU die grenzüberschreitende Zusammenarbeit zwischen den Finanzaufsichtsbehörden verstärkt werden. Gleichzeitig ist die Aufsicht über Institute, Märkte und Produkte so zu erweitern, dass Gewerkschaften, Verbraucherschützer, Nichtregierungsorganisationen usw. beteiligt werden.

Teil einer neuen internationalen Finanzarchitektur, eines neuen Bretton Woods, sollte auch die Regulierung der Wechselkurse und kurzfristig orientierter Kapitalströme sein. Zur Überwachung und Durchsetzung einer solchen neuen Ordnung brauchen wir auch neue internationale Institutionen. Der IWF ist durch seine jahrzehntelange Politik neoliberaler Strukturanpassungsprogramme diskreditiert und hierfür nicht geeignet.

Aber nicht nur die Kontrolle, auch die Rolle der Banken muss neu bestimmt werden. Investmentbanking, die Erfindung immer neuer Produkte - alles dies hat zur Krise geführt. Banken sind ihrer Funktion nach Kapitalsammelstellen, die die Verbraucher und die Unternehmen mit Liquidität versorgen sollen. Sie im Wesentlichen auf diese Aufgabe zu beschränken, diese Orientierung muss der Regulierung der Kapitalmärkte zugrunde gelegt werden.

Neben der verschärften Regulierung sollte die Stärkung des staatlichen Einflusses in einer größeren Rolle öffentlicher Banken zum Ausdruck kommen. Sparkassen und Genossenschaftsbanken sollen gestärkt werden. Auch die Verstaatlichung privater Banken gehört in der heutigen Situation auf die Tagesordnung. Allerdings muss, das hat das Finanzgebaren der Landesbanken gezeigt, neben die Kontrolle durch öffentliches Eigentum eine reale Kontrolle der Aktivitäten und ein am öf- fentlichen Auftrag orientiertes Geschäftsmodell treten.

\section{WENDE ZU SOZIALSTAATLICHER POLITIK}

Grundlagen der jahrelang bestehenden Überliquidität der Finanzmärkte waren erstens die Umverteilung zugunsten der Kapital- und Vermögensbesitzer über Steuersenkungen und der Rückgang der Lohnquote angesichts von Massenarbeitslosigkeit und einer geschwächten gewerkschaftlichen Verhandlungsposition. Zweitens hat auch die Teilprivatisierung der Rentensysteme im globalen Maßstab Billionensummen auf die Finanzmärkte umgeleitet. Drittens wurden vormals öffentliche Unternehmen wie etwa die Telekom über den Kapitalmarkt privatisiert.

Wir benötigen daher eine neue Form gerechter Besteuerung, damit das Problem der Überliquidität der Finanzmärkte behoben werden kann. Gleichzeitig würden Geld und Kapital in die öffentliche Hand umgelenkt und könnten für dringend benötigte Investitionen verwendet werden.

Um der Krise zu begegnen, muss der Staat über ein schnell wirkendes Programm zur Konjunkturstabilisierung hinaus ein Zukunftsinvestitionsprogramm für Arbeit, Bildung und Umwelt mit zusätzlichen öffentlichen Ausgaben von mindestens $100 \mathrm{Mrd}$. $€$ jährlich auflegen. Diese Summen und die dadurch ausgelösten Investitionen würden eine doppelte Wende einleiten: Sie fördern ein qualitatives Wachstum, stärken die für eine funktionierende Volkswirtschaft notwendige Binnennachfrage und sie schaffen, auf der Basis sozial gerechter und ertragreicher Steuern, auf Dauer ein neues Fundament des Sozialstaates. Dieser Prozess antizyklischer Stabilisierung wird über eine Neuverschuldung finanziert werden müssen. Sie darf nicht durch eine monetaristische Politik des starken Euro oder das Dogma des schuldenfreien Haushaltes - einer im Grundgesetz verankerten Schuldenbremse - behindert werden.

Auch im Bereich der sozialen Sicherheit benötigen wir verstärkte staatliche Anstrengungen, um Armutsgefahren zu begegnen und Teilhabe am gesellschaftlichen Leben zu ermöglichen.

Da sich unser soziales Sicherungssystem auf die Erwerbsarbeit stützt, ist der gesetzliche Mindestlohn von 7,50 € ebenso unabdingbar wie die Allgemeinverbind- 
lichkeit von Tarifverträgen, die über diesem Mindestlohn liegen, damit sie in bestimmten Branchen nicht durch Lohnund Sozialdumping durch die Arbeitnehmerfreizügigkeit und das europäische Wettbewerbsrecht ausgehebelt werden können.

Dazu gehören auch die Eingrenzung der Leiharbeit nicht nur durch den Mindestlohn, sondern durch das Prinzip gleicher Lohn für gleiche Arbeit und das Verbot, Leiharbeiter befristet nur für einen Job einzustellen.

Das öffentliche, umlagefinanzierte Rentensystem muss gegenüber den privaten, kapitalgedeckten Systemen wieder gestärkt werden. Denn Kürzungen des Rentenniveaus, Niedriglöhne, Arbeitslosigkeit und die geringe Sparfähigkeit einkommensschwacher Haushalte haben die Gefahr der Altersarmut erhöht.

Wir brauchen eine Erweiterung der gesetzlichen Rentenversicherung $\mathrm{zu}$ einer allgemeinen Erwerbstätigenversicherung, um eine Antwort auf die zunehmend unterbrochenen Erwerbsbiografien und die wachsende Zahl von Selbstständigen und Freiberuflern zu finden.

Armut geht immer mehr mit Ausgrenzung einher. Darum darf an den sozialen Diensten, an der Bildung und am sozialen Wohnungsbau nicht gespart werden. Im Gegenteil: Nur wenn wir Millionen aus der
Armut holen und ihnen neue Bildungschancen eröffnen, werden wir ihnen eine gerechte Teilhabe am gesellschaftlichen Leben ermöglichen. Auch das ist mehr als eine zukünftige Aufgabe des Staates.

Und nicht zuletzt: Von der Grundsicherung nach Hartz IV kann niemand leben. Darum brauchen wir eine Erhöhung auf $420 €$. Diese Erhöhung wird auch einen Beitrag zur Stimulierung der Binnennachfrage liefern, da die Bezieher niedriger Einkommen nicht in der Lage sind, Konsumverzicht zu üben.

\section{AUSBAU ÖFFENTLICHER DIENSTLEISTUNGEN}

Nach unserem Verständnis umfasst der Sozialstaat mehr als die soziale Absicherung gegen Krankheit, Alter und Arbeitslosigkeit. Sozialstaat bedeutet auch öffentliche Dienstleistungen. Marktradikale Politik in Deutschland wie in Europa hat gerade den öffentlichen Sektor durch eine Deregulierung, Liberalisierungs- und Privatisierungswelle in seinen Grundfesten erschüttert. Dabei wurden viele der erwünschten Effekte - besserer Service, niedrige Preise, mehr Bürgerfreundlichkeit - nicht wie versprochen erreicht. ${ }^{3}$

Dem Ausverkauf öffentlicher Dienstleistungen müssen wir einen Riegel vor- schieben. Das EU-Recht sieht ausdrücklich vor, dass der öffentliche Sektor nach dem Subsidiaritätsprinzip Aufgabe der Mitgliedstaaten ist, und auch der Vertrag von Lissabon betont dieses Recht der Mitgliedstaaten, der regionalen wie der lokalen Behörden. Eine sozialstaatliche Wende muss daher auf diesem Prinzip beharren. Natürlich müssen in allen europäischen Ländern öffentliche Dienste auf hohem Niveau erbracht und angeboten werden. Aber gegen ein Europa, das dieses Niveau durch Liberalisierung und Privatisierung absenkt, brauchen wir eine Politik der Wiederaneignung öffentlichen Eigentums und öffentlicher Verfügungsmacht. Öffentliches Eigentum, öffentliche Verfügungsmacht über die Daseinsvorsorge und Daseinsfürsorge der Menschen ist wesentlich für die Lebensqualität und damit ein Leben in Würde.

Auf den Punkt gebracht: Die künftigen Aufgaben des Staates bestehen vor allem darin, sich wieder seines Auftrages und seiner Aufgaben als Sozialstaat unseres Grundgesetzes zu besinnen und danach zu handeln.

\section{Gewerkschaftliche Eckpunkte einer aktiven Industriepolitik}

Mit der Bestimmung einer aktiven Industriepolitik ist der Anspruch verbunden, den industriellen Sektor in Deutschland zu gestalten und sich nicht mit der passiven Anpassung an einen Wandel der wirtschaftlichen Rahmenbedingungen zu begnügen. Damit ist bereits eine grobe inhaltliche Richtung vorgegeben, die in dem vorliegenden Beitrag zu einer Skizze grundlegender industriepolitischer Gestaltungsansätze und Strategien aus gewerkschaftlicher Sicht konkretisiert wird.

\section{BEDEUTUNG UND FORMWANDEL DES INDUSTRIELLEN SEKTORS}

Zunächst einmal gilt es festzustellen: Form, Inhalt und Tempo des industriellen Strukturwandels und der industriellen Entwicklung bestimmen nachhaltig die Arbeitsund Lebensbedingungen der Beschäftigten im industriellen Sektor - und nicht nur dort. Der industrielle Sektor ist - jenseits aller Debatten um eine Dienstleistungsund Wissensgesellschaft - nach wie vor die Basis der ökonomischen Entwicklung unserer Gesellschaft.
Dieser Befund lässt sich durch wenige Zahlen untermauern. Laut einer jüngst im Auftrag des Büros für Technikfolgenabschätzung beim Deutschen Bundestag veröffentlichten Studie entfallen etwa $90 \%$ der Forschungs- und Entwicklungsaufwendungen der deutschen Wirtschaft auf

\footnotetext{
Martin Allespach, PD Dr., ist Bereichsleiter für Grundsatzfragen, Gesellschaftspolitik und strategische Planung beim Vorstand der IG Metall. e-mail: martin.allespach@igmetall.de
} 
das verarbeitende Gewerbe. ${ }^{1}$ Daneben haben die produzierenden Unternehmen eine Schrittmacherfunktion als wichtiger Nachfrager und Impulsgeber für sogenannte produktionsnahe Dienstleistungen, insbesondere Datenverarbeitung und Datenbanken, Forschung und Entwicklung sowie Erbringung von Dienstleistungen für Unternehmen. Zusammen mit diesen Sektoren beschäftigt das verarbeitende Gewerbe fast $60 \%$ der Arbeitnehmerinnen und Arbeitnehmer in Deutschland und erwirtschaftet fast $80 \%$ der Produktionswerte.

Somit ist kein ökonomischer Bedeutungsverlust des industriellen Sektors zu konstatieren, sondern ein beschleunigter Strukturwandel, der sich durch neuartige Verbünde von traditioneller Industrieproduktion und industrienahen Dienstleistungen auszeichnet und unter dem Stichwort ,interne Tertiarisierung “ Eingang in die Fachdiskussion gefunden hat.

Vor diesem Hintergrund besteht keinerlei Anlass zu einer Abkehr von industriepolitischen Aktivitäten oder gar zu einem Rückzug aus der Industriepolitik. Das Gegenteil ist notwendig: Es gilt, die Auseinandersetzung über die zentrale Bedeutung der Industriepolitik zu forcieren und dabei den Veränderungen des industriellen Sektors Rechnung zu tragen - auch in konzeptioneller Hinsicht, z. B. bei der Formulierung von industriepolitischen Gestaltungsanforderungen. Letztere müssen - um nachhaltig Wirkung entfalten zu können auch Bezug nehmen auf wirtschaftliche Entwicklungen, die ganz maßgeblich die Richtung und das Tempo der industriellen Entwicklungen bestimmt haben. In diesem Sinne ist eine arbeitnehmer- und innovationszentrierte aktive Industriepolitik voraussetzungsvoll; sie steht vor drei zentralen Herausforderungen: Finanzmarktkapitalismus mit Shareholder-Value-Orientierung, Unternehmensstrategien mit Kostensenkungsfokus, Internationalisierung mit grenzüberschreitenden Wertschöpfungsketten.

\section{FINANZMARKTKAPITALISMUS}

Der Finanzmarktkapitalismus hat die Dominanz des Shareholder-Value-Prinzips mit dessen inhärenter Orientierung an kurzfristigen Unternehmenszielen befördert. Als Folge unterblieben auch notwendige Investitionen und Innovationen in den Betrieben, die im Sinne einer langfristig nachhaltigen industriellen Entwicklung erforderlich (gewesen) wären. So klafft laut einer Studie des Instituts für Makroökonomie und Konjunkturforschung (IMK) ${ }^{2}$ eine immer stärkere Lücke zwischen Investitionen und Unternehmensgewinnen. In diesem Zusammenhang gilt es, auf eine bedrohliche Entwicklung hinzuweisen: Das Produktivitätswachstum in Deutschland liegt im unteren Drittel der OECD-Länder und damit deutlich unter dem Durchschnitt. Das heißt nichts anderes, als dass Deutschland seine Weltmarkterfolge in den letzten 20 Jahren vor allem durch eine Lohnmoderation und nicht durch Produktivitätszuwächse errungen hat. Diese „Strategie" ist aber nicht nachhaltig, weil beim dauerhaften Verzicht auf Produktivitätswachstum als zentraler Wettbewerbsparameter nur die Lohnhöhe übrig bleibt. Dann aber ist der Weg vom Gestalter zum Getriebenen des Marktgeschehens extrem kurz.

Wirtschafts- und industriepolitische Ansatzpunkte zur Begrenzung der mit dem Shareholder-Value-Prinzip verbundenen Probleme müssen an den institutionellen Rahmungen ansetzen, die zur Herausbildung seiner Dominanz geführt haben: an der Liberalisierung und Deregulierung der Finanzmärkte. Ohne eine striktere Regulierung der Finanzmärkte, ohne Aufhebung ihrer Dominanz über Realwirtschaft und Politik, lässt sich auf Dauer keine nachhaltige, d. h. ökologisch und sozial verträgliche industrielle Entwicklung durchsetzen.

\section{UNTERNEHMENSSTRATEGIEN}

Eine nachhaltige Industriepolitik setzt auf Qualitäts- und nicht auf Kostensenkungsstrategien („besser statt billiger"). ${ }^{3}$ Gute Arbeit, Innovationen und gute Bildung sind die Grundlage unseres Produktionsmodells und Voraussetzungen für dessen Erfolg. Unternehmen hingegen, die ihre Marktstellung über Lohndumping, Tarifverzicht und Arbeitszeitausweitung behaupten, verspielen ihre Zukunft. Ihre Arbeitsplätze sind die ersten, die vom Markt verschwinden, weil andere diese anspruchslose Strategie noch radikaler umsetzen können. ${ }^{4}$ Erfolgreicher sind dagegen diejenigen Unternehmen, die auf Innovationen - auf neueste Technologien, innovative Produkte, die Beteiligung und Mitbestimmung der Beschäftigten - setzen. Denn die Innovationsfähigkeit deutscher Industriebetriebe ist eine der entscheidenden Determinanten für die Sicherung der zukünftigen Wettbewerbsfähigkeit und Wertschöpfung in Deutschland. Unternehmen müssen nach der richtigen Innovationsstrategie suchen und haben dabei mehr Wahlmöglichkeiten, als vielfach behauptet wird.

Neben den klassischen (techniklastigen) Forschungs- und Entwicklungsbereichen ist betriebliches Wachstum auch durch verbesserte Dienstleistungen oder Organisationsinnovationen möglich. Um diese Potenziale zu erschließen, müssen die Beschäftigten, die betrieblichen Praktiker, die betrieblichen Interessenvertreter und die Gewerkschaften einbezogen werden, als Experten in eigener Sache. Auf den ersten Blick mag das Thema "besser statt billiger“ quer zu den klassischen industriepolitischen Fragestellungen liegen. Gleichwohl besteht ein untrennbarer Zusammenhang, da Industriepolitik ein Querschnittsthema ist, dessen erfolgreiche Bearbeitung nicht zuletzt davon abhängt, inwieweit wichtige Themenfelder wie gute Arbeit, gute Bildung und Innovationspolitik in die Gesamtstrategie eingebunden werden. HighRoad-Produkte und Low-Road-Arbeitsbedingungen schließen sich aus. Mit anderen Worten: Der Erfolg deutscher Maschinenund Anlagenbauer ist weder Zufall noch gottgegeben, sondern Resultat eines spezifischen (und verletzlichen) Produktionsregimes, dessen zentrale Bestandteile erst in der Summe ihre volle Wirkung entfalten. ${ }^{5}$

\section{INTERNATIONALISIERUNG}

Über Fusionen und Übernahmen, aber auch über den Aufbau neuer Produktionsstätten in anderen Ländern, sind in den vergangenen Jahrzehnten etliche Unternehmen entstanden, deren Aktivitätsschwer-

\footnotetext{
1 Kinkel, S./Friedewald, M./Hüsing, B./Lay, G./ Lindner, R. (2008): Arbeiten in der Zukunft. Strukturen und Trends der Industriearbeit, Berlin.

2 Hein, E./van Treek, T. (2008): Finanzmarktorientierung - ein Investitions- und Wachstumshemmnis?, IMK-Report 26, Düsseldorf.

3 Kampagne der IG Metall Bezirksleitung NordrheinWestfalen bis 2007 (vgl. Mitbestimmung 11/2005, S. 10-19).

4 Auch volkswirtschaftlich führt eine Kostensenkungsstrategie wegen des Kaufkraftentzuges in eine Sackgasse. Wie sich in der gegenwärtigen Krise zeigt, ist die Nachfrageseite die Achillesferse der deutschen Volkswirtschaft, und nicht die vermeintlich zu hohen Produktionskosten.

$5 \quad$ Hall, P. A./Soskice, D. (Hrsg.) (2001): The Institutional Foundations of Comparative Advantage, Oxford.
} 
punkt in ganz Europa bzw. weltweit liegt und die in Europa maßgeblich zu der fortgeschrittenen ökonomischen Integration der Europäischen Union beigetragen haben. Damit verbunden war und ist die Herausbildung grenzüberschreitender Wertschöpfungsketten, eine Entwicklung, die insbesondere die Automobilindustrie prägt. So haben fast alle europäischen Konzerne und die europäischen Teile der US-Autokonzerne Produktionsstandorte in verschiedenen europäischen Ländern, in denen Aggregate oder Komponenten für andere Standorte produziert werden. Die Kapitalseite ist in diesen Fällen in der Regel global oder europäisch aufgestellt, das heißt, Investitions-, Produktions- und Standortentscheidungen wie auch Restrukturierungsprogramme setzen europaweit an. Entsprechend lässt sich Industriepolitik heute nur im europäischen Kontext gestalten, zumal wesentliche (wettbewerbs-)rechtliche Grundlagen für eine aktive staatliche Intervention ohnehin auf diese Ebene verlagert wurden. Das hindert einzelne Länder allerdings bisher nicht daran, „nationalen“ Interessen im Konfliktfall den Vorzug zu geben. Während beispielsweise Frankreich nach wie vor nationale Champions direkt und indirekt begünstigt, setzt die deutsche Regierung in weitaus stärkerem Maße auf die vermeintlichen Segnungen des freien Spiels der Marktkräfte. Auf längere Sicht sind nationale Alleingänge innerhalb eines hochintegrierten Wirtschafts- und Währungsraums problematisch, da Renationalisierungstendenzen den Wettbewerb verzerren und Desintegration auf der politischen Ebene befördern.

Notwendig ist vielmehr eine europaweit koordinierte aktive Industrie- und Strukturpolitik, die zweierlei leisten muss: den Erhalt und Ausbau der industriellen Kerne in den alten EU-Mitgliedstaaten und die Unterstützung des industriellen Aufholprozesses in den neuen EU-Mitgliedstaaten und unterentwickelten Regionen. Dabei gilt es, eine europäische Wettbewerbsordnung zu implementieren, die bei aller Notwendigkeit der Berücksichtigung der verschiedenen volkswirtschaftlichen Entwicklungsstadien - nicht dazu führt, dass die Arbeitsbedingungen innerhalb einer Branche in eine Abwärtsspirale geraten. In diesem Zusammenhang sind auch die Gewerkschaften gefordert, die Branchenarbeit auf europäischer Ebene weiter voranzutreiben und die Handlungsarena der europäischen Betriebsräte besser zu nutzen. Grundsätzlich muss aber gelten: Unternehmen konkurrieren miteinander, nicht einzelne Länder. Wer dies nicht berücksichtigt, ist dem Droh- und Druckpotenzial der Unternehmen ausgesetzt.

\section{WIRTSCHAFTS- UND GESELL- SCHAFTSPOLITISCHE OPTIONEN}

Richtung und Tempo industrieller Entwicklung werden schließlich auch bestimmt durch wirtschafts- und gesellschaftspolitische Optionen - wie die Dominanz neoliberaler Politik oder aktive industriepolitische Entwicklungsstrategien. Dabei gilt es immer wieder zu betonen, dass es Alternativen zum schlichten reaktiven Management einer erzwungenen Anpassung an vermeintliche Imperative der Standortsicherung gibt, dass der marktwirtschaftliche Selbstlauf - wie ihn das neoliberale Credo vorsieht - keineswegs zu Ergebnissen führt, die aus Sicht der Beschäftigten akzeptabel sind. Massenarbeitslosigkeit, Strukturbrüche, Vergeudung gesellschaftlicher und natürlicher Ressourcen, Umweltbelastungen sowie Produktions- und Standortverlagerungen unter rein profitorientierten Gesichtspunkten sind keine Naturgesetze und stehen im Widerspruch zu einer sozial regulierten Ökonomie und lebendigen Demokratie.

Es geht entsprechend um die Notwendigkeit der Wiedererlangung des Primats der Politik gegenüber der Ökonomie als konstitutives Element von Demokratie. Unter der Überschrift, „Demokratiealarm“ hat Heribert Prantl einen Kommentar zur Finanzmarktkrise geschrieben. Dort heißt es:

„Die Großmanager des Geldmarkts taten so, als sei die Demokratie eine Spielwiese für Kleinbürger, und als hätten Wahlkämpfe und Wahlen nur eine Funktion ähnlich der, wie sie „Brot und Spiele“ im alten Rom hatten - weil die wahren Wahlakte der Überzeugung der Großmanager nach auf dem Börsenmarkt stattfanden. ... Rolf Breuer, der frühere Vorstandschef der Deutschen Bank legte einst dar, warum sich Regierungen nach den Wünschen der Anleger richten müssten: Die autonomen Entscheidungen, die Hunderttausende von Anlegern auf den Finanzmärkten treffen, werden im Gegensatz zu den Wahlentscheidungen nicht alle vier oder fünf Jahre, sondern täglich gefällt. ... Die demokratische Kontrolle der Regierungen durch die Bürger würde so überflüssig, weil nach dieser Philosophie die freien Finanzmärkte die
Politik wirkungsvoller kontrollieren, im Grunde sogar ersetzen konnten.... Demokratie ist (aber ihrem Wesen nach) eine Gemeinschaft, die ihre Zukunft miteinander gestaltet. Die Dirigenten der Finanzmärkte haben sich diesem Miteinander" weitgehend entzogen. Nun hängt es von der Politik ab, diese Entwicklung zu revidieren, Regularien für die Märkte durchzusetzen und effektiv zu kontrollieren bzw. Missachtung $\mathrm{zu}$ sanktionieren (Süddeutsche Zeitung von 9.10.2008).

Was Heribert Prantl hier für die Regulation der Finanzmärkte einfordert, gilt genauso für die Industriepolitik. Auch an der Notwendigkeit einer gesellschaftlichen Einflussnahme auf die industrielle Entwicklung und einer gesellschaftlichen Gestaltung des industriellen Strukturwandels besteht kein Zweifel. Es bleibt zu fragen: An welchen Kriterien sollte sich eine solche Einflussnahme und Gestaltung ausrichten? Und wie lassen sich unsere gewerkschaftlichen Eckpunkte zusammenfassen?

\section{EIN INDUSTRIEPOLITISCHES LEITBILD AUS SICHT DER GEWERKSCHAFTEN}

Eine ausschließlich defensive Anpassung an Trends der Kapitalakkumulation und dominante Entwicklungsmuster scheidet als Orientierungspunkt aus, auch wenn es selbstverständlich ist, dass industriepolitische Gestaltungsansätze und Strategien immer die realen Kontexte berücksichtigen müssen. Ebenfalls kann es aus Sicht einer Industriegewerkschaft nicht darum gehen, die eigenen industriepolitischen Optionen ausschließlich an den Interessen einzelner Unternehmen oder einzelner Branchen auszurichten, seien Letztere auch noch so dominant wie z. B. die Automobil- oder die Chemieindustrie. Gesellschaftliches Leitbild für eine aktive Industriepolitik aus gewerkschaftlicher Sicht ist das Kriterium der Nachhaltigkeit.

Nachhaltig ist die industrielle Entwicklung zunächst dann,

- wenn sie unter gesamtwirtschaftlichen Gesichtspunkten ökonomisch effizient ist; - wenn sie sozial tragfähig ist - also strukturellen Wandel ohne Brüche bei der Beschäftigung und den Einkommen gewährleistet und zur Sicherung einer regionalen Ausgewogenheit beiträgt,

- wenn sie ökologisch verträglich gestaltet ist und einen effizienten und umweltschonenden Ressourceneinsatz erreicht. 
Diese Punkte müssen aus Sicht der IG Metall um ein weiteres konstitutives Nachhaltigkeitskriterium ergänzt werden: Unerlässlich ist eine umfassende demokratischgesellschaftliche Beteiligung durch institutionalisierte Beteiligungs- und Mitbestimmungsrechte der Beschäftigten sowie ihrer Gewerkschaften bei wesentlichen wirtschaftlichen Entscheidungen. Weil es nicht darum geht, einen Optimalzustand auf dem Gesetzesweg zu verordnen und $\mathrm{zu}$ konservieren, sondern im Rahmen einer sozial regulierten Marktwirtschaft ständig neue Herausforderungen zu bewältigen. Funktionale sowie auf breiter Legitimationsbasis aufsetzende Lösungen sind aber nur dann möglich, wenn allen Anspruchsgruppen (Stakeholdern) weitreichende Mitwirkungsrechte eingeräumt werden. Nicht hinzunehmen ist - um ein konkretes Beispiel anzuführen - eine Entwicklung wie in der ostdeutschen Fotovoltaikindustrie, ${ }^{6}$ die mit erheblichem Einsatz öffentlicher Mittel gefördert wurde, eine weitgehend mitbestimmungs- und gewerkschaftsfreie Zone darstellt, selbst im ostdeutschen Vergleich niedrigste Löhne zahlt, alle gesetzlich möglichen Ausnahmeregelungen bezüglich der Arbeitszeiten extensiv ausnutzt und die Mitgliedschaft im Arbeitgeberverband scheut.

Die umfassenden Anforderungen einer nachhaltigen Entwicklung bedeuten, dass die Umsetzung dieser Ziele vielfach einer Gratwanderung zwischen unterschiedlichen - sich manchmal widersprechenden Anforderungen gleichkommt. Diese gilt es auszutarieren. Wie dies konkret geschehen kann, lässt sich an den (teilweise in das Konjunkturpaket II eingeflossenen) Forderungen der IG Metall zur Konjunkturbelebung und Arbeitsplatzsicherung in der Automobilindustrie veranschaulichen.
Dazu nur ein Schlaglicht: Die IG Metall hat Forderungen aufgestellt, die den Grundsätzen von Nachhaltigkeit mit ihren ökologischen, ökonomischen, gesellschaftlichen, sozialen und demokratiepolitischen $\mathrm{Di}$ mensionen entsprechen. Dazu gehören unter anderem:

(1) Eine Umweltprämie für die Verschrottung von Altautos, also Fahrzeuge, die nicht dem heutigen Stand von CO2-Ausstoß und Kraftstoffverbrauch genügen (ökologische Wirkung).

(2) Ein Zukunftsinvestitionsprogramm „Arbeit, Bildung und Umwelt" als Investition für Kommunen und Länder in Bildung, Forschung und Umwelt sowie Kredite zum EZB-Zins an Unternehmen, wenn sie die Energie- und Ressourceneffizienz nachhaltig steigern (gesamtgesellschaftliche, ökologische und ökonomische Wirkung).

(3) Eine staatliche Förderung für Einzelunternehmen soll es nur gegen eine Ausweitung der Mitbestimmungsrechte geben (demokratiepolitische Dimension), z. B. eine Neuregelung im Mitbestimmungsgesetz, wonach die Errichtung, Verlegung oder Schließung von Produktionsstätten der Zustimmung des Aufsichtsrats bedarf. Für diese Zustimmung muss eine Mehrheit von zwei Drittel der Mitglieder des Aufsichtsrats erforderlich sein, wie dies bei VW der Fall ist.

Etliche der genannten Punkte hätten sicherlich einer ausführlicheren Darstellung und einer weiteren Konkretisierung bedurft. Wichtige Themen wie z. B. der Ausbau der Forschungsinfrastruktur, Maßnahmen zur Sicherstellung des Informations- transfers, Initiativen zur Clusterbildung, die Erhöhung der Durchlässigkeit des Bildungssystems etc. wurden vollständig ausgeblendet, weil es in diesem Beitrag darum ging, jenseits aller Detailvorschläge die wichtigsten Forderungen aus Sicht der IG Metall zu skizzieren.

Zusammenfassend lässt sich festhalten: Erstens ist Industriepolitik kein altes Thema, das sich überholt hat. Zweitens gilt es, Industriepolitik zu gestalten. Dafür müssen wir Ansätze im Sinne der Nachhaltigkeit entwickeln und in konkrete nationale, europäische und internationale Politik umsetzen. Drittens ist Industriepolitik kein isolierter Politikbereich. Sie hat sich den ökologischen, gesamtgesellschaftlichen und demokratiepolitischen Herausforderungen offensiv zu stellen. Viertens ist es industriepolitisch nicht ausreichend, bestimmte Maßnahmenpakete zur Stärkung der Industrie aufzulegen, sondern in gleichem Maße ist dafür Sorge zu tragen, dass der betriebliche Alltag Innovation zulässt und befördert.

Es bleibt die Hoffnung, dass gerade in Zeiten tiefer wirtschaftlicher Verwerfungen diese grundlegenden Zusammenhänge neu entdeckt und mit Leben gefüllt werden. Anders formuliert: Um einen wirklichen Pfadwechsel einzuleiten, bedarf es einer Krise des Bestehenden und realistischer Alternativen. An ihrer Ausformulierung (und Umsetzung) können und werden die Gewerkschaften aktiv mitarbeiten.

6 Richter, U./Horst, G./Krippendorf, W. (2008): Solarindustrie als neues Feld industrieller Qualitätsproduktion - das Beispiel Photovoltaik, OBS Arbeitsheft 56, Frankfurt/Main. 